\title{
Introduction to the Special Issue on Individual Differences in Multisensory Perception: An Overview
}

\author{
Clare Jonas $^{1, *}$, Mary Jane Spiller ${ }^{1}$, Paul B. Hibbard ${ }^{2}$ and Michael Proulx ${ }^{3}$ \\ ${ }^{1}$ School of Psychology, University of East London, Stratford Campus, Water Lane, London \\ E15 4LZ, UK \\ ${ }^{2}$ Department of Psychology, University of Essex, Wivenhoe Park, Colchester, CO4 3SQ, UK \\ ${ }^{3}$ Department of Psychology, University of Bath, Claverton Down, Bath, BA2 7AY, UK \\ * To whom correspondence should be addressed. E-mail: clare.n.jonas@gmail.com
}




\begin{abstract}
The world is full of objects that can be perceived through multiple different senses to create an integrated understanding of our environment. Since each of us has different biological and psychological characteristics, different people may perceive the world in quite different ways. However, the questions of how and why our multisensory perceptions differ have not been explored in any great depth.

This special issue, arising from a series of British Psychological Society-funded seminars, presents new research and opinions on the impacts of a variety of individual differences on multisensory perception. We hope that readers will enjoy this collection of eight papers on individual differences in multisensory perception arising from developmental changes, autism, Down syndrome, migraine, sensory loss and substitution, and personality.
\end{abstract}

Keywords: individual differences, developmental changes, autism, Down syndrome, migraine, blindness, personality 
In the history of the psychology of human perception, much of the research carried out has been on one sense at a time. This research concentrated largely on vision, as the dominant sense in humans, and to a lesser extent on hearing and somatosensation, while the chemical senses were relatively neglected (Smith, 2015). However, over the last 25 or so years, human perception research has gradually changed to consider multisensory perspectives (Alais et al., 2010), to the extent that early sensory processing is now considered to be fundamentally multisensory rather than unisensory (Ghazanfar and Schroeder, 2006). We are now in a position to consider how multisensory processing might differ between groups and between individuals - an important question for our understanding of neurodivergent conditions, ageing, and sensory loss. It was with this question in mind that we ran a series of British Psychological Society-funded seminars that have now become the basis for this special issue.

Some of the work of mapping out individual differences in multisensory processing has already begun, but many areas of inquiry remain. We can broadly divide categories of individual difference into two areas, longitudinal and cross-sectional.

Longitudinal differences are relatively well-explored. We know that an infant's multisensory world is quite different from that of a young adult (e.g. Lewkowicz and Ghazanfar, 2009), and that multisensory perception in young adults will change over the lifespan, whether they remain healthy into old age or develop pathologies (e.g. Chan et al., 2014; Setti et al., 2011). In the current special issue, two papers investigate novel aspects of such longitudinal changes.

Greenfield, Ropar, Themelis, Ratcliffe and Newport (this issue) assessed the effect of spatial and temporal discrepancies between space and touch. They found that asynchronous stimuli were less likely to be integrated by 11-year-olds than 4-year-olds, supporting the idea that multisensory integration is spatially and temporally refined over the course of development.

In their review of vicarious tactile perception, Gillmeister, Bowling, Rigato and Banissy (this issue) present evidence for vicarious touch both in infants and in older adults, arguing that the early developmental origins of our inter-individual differences highlight the importance of studying multisensory perception across the entire lifespan. They also take a cross-sectional approach, reviewing evidence relating to vicarious touch in both neurotypical individuals and to those who experience a conscious sensation of touch on 
their own body from viewing touch to another person (known as mirror-touch synaesthesia).

Cross-sectional individual differences in multisensory perception are less wellexplored; the focus here has largely been on differences between the general population and those with benign or harmful neurological and psychological conditions such as synaesthesia (e.g. Lacey et al., 2016), schizophrenia (e.g. Szycik et al., 2009; Williams et al., 2010), and specific learning impairments (Kaganovich et al., 2014). Four papers explore the area of neurological conditions in this special issue.

Like schizophrenia and synaesthesia, autism spectrum disorder (ASD) is well known to involve unusual multisensory processing styles. For example, the temporal binding window appears to be longer in children with ASD than in neurotypical children (Foss-Feig et al., 2010); young children with ASD may also have trouble integrating the auditory and visual aspects of speech (Foxe et al., 2015). Poole, Poliakoff, Gowen, Couth, Champion and Warren (this issue) compared the integration of visual and haptic information in ASD and neurotypical controls, and whether performance in the two groups could be predicted by a statistically optimal maximum likelihood estimation (MLE) model (Ernst and Banks, 2002). Poole et al. hypothesised that the atypical neuronal development in ASD might have an impact on the maturation of visuo-haptic integration. Their results showed no difference in the way in which the two groups integrated information. However, performance for both groups was more consistent with a cue-switching model than the MLE model. Since the MLE model has been found to be a good predictor of performance in previous studies (e.g. Gori et al., 2008), Poole et al. discuss a range of individual differences which might contribute to this discrepancy such as participants' expectations about whether cues should be combined and level of experience with psychophysical observations, which might both have influenced the strategy they adopted.

Hamburg, Startin and Strydom (this issue) have explored sound-shape matching, as an indicator of multisensory integration (MSI) in individuals with Down syndrome (DS). Using the well-known bouba-kiki paradigm, they found that although sound-shape matching deficits in DS might be relatively common, they are likely related to lower cognitive ability, rather than a general characteristic of DS.

Much less is known about multisensory perception in migraine than the other disorders so far covered; O'Hare (this issue) draws together the existing knowledge on 
several areas of multisensory integration in migraine and offers some interesting new directions that research in this area may take.

One factor that potentially contributes to the multisensory processing differences explored in this review is that there might be longer temporal integration of sensory signals in migraine. The physiological correlate of this individual differences is explored in detail by Keil and Senkowski (this issue), who show that variation in the temporal integration window of the sound-induced flash illusion (Shams et al., 2000) is related to the frequency of alphaband oscillation (Cecere et al., 2015). They also demonstrate that activity in the visual cortex contributes to this relationship.

In addition to the neurological conditions outlined above, multisensory perception is now being explored as a feature of a number of physiological conditions such as visual field defects and unilateral spatial neglect (Bolognini et al., 2016), Parkinson's disease (Ding et al., 2017), functional movement disorders (Marotta et al., 2017), and stroke (White and Aimola Davies, 2017). The most highly-investigated physiological conditions are blindness and deafness, and in this special issue Arnold, Pesnot-Lerousseau and Auvray review how individual capacities impact the ability to use assistive technology for the visually impaired. A special type of assistive technology called a sensory substitution device transforms the missing visual input into a format that another intact sensory modality can process. For example, images can be turned into touch on the tongue (Bach-y-Rita and Kercel, 2003) or as auditory displays (Meijer, 1992). Tantalizing clues that individual differences in auditory abilities correlate with performance using an auditory display, the vOICe, suggested that prior musical experience afforded enhanced perceptual or cognitive abilities to assist in learning to use this new technology (Haigh et al, 2013). The review by Auvray and colleagues concludes that the individual differences approach would be a fruitful one for revealing the mechanisms involved in learning to see by hearing or touch.

The final cross-sectional difference covered in this paper is a novel one personality. Autonomous Sensory Meridian Response (ASMR) is a pleasant, tingling sensation that is triggered in some people by specific auditory and visual stimuli (Barratt and Davis, 2015). Janik McErlean and Banissy (this issue) showed that those who experience ASMR tend to score higher in openness to experience, empathic concern and fantasizing, and lower in conscientiousness, than a control group. This personality profile bears some similarity with that found in synaesthesia (Banissy et al, 2013), and indeed the prevalence of 
self-reported synaesthesia in those who experience ASMR is relatively high (Barratt and Davis, 2015).

This special issue shows the importance of considering differences in multisensory perception across the lifespan and also between groups, such as those with migraines, ASD, Down syndrome or sensory impairment. The implications within the population such as neural activity or personality have also been considered. Taste and smell have not been covered in this issue, reflecting the general emphasis of multisensory research on vision, hearing and touch; the chemical senses are an important area for future research on individual differences.

To a large degree, individual differences in perception have been ignored, and the small numbers of participants used in many psychophysical studies mean that data on these differences are simply not available in most cases. Peterzell and Kennedy (2016), in presenting a 'factor analytical manifesto', argue that, rather than a source of error to be dismissed, individual differences allow greater theoretical understanding of perception, insight regarding the typical and abnormal development of perceptual systems, and for links to be made between behavioural and neurophysiological measures. The papers presented in this special issue demonstrate clear examples of all of these in action in the realm of multisensory perception, and the practical potential that the greater depth of understanding of conditions such as migraine and ASD provides.

\section{References}

Alais, D., Newell, F. N. and Mamassian, P. (2010). Multisensory processing in review: from physiology to behaviour, Seeing Perceiving 23, 3-38.

Bach-y-Rita, P. and Kercel, S. W. (2003). Sensory substitution and the human-machine interface, Trends Cogn. Sci. 7, 541-546.

Banissy, M. J., Holle, H., Cassell, J., Annett, L., Tsakanikos, E., Walsh, V., Spiller, M. J. and Ward, J. (2013). Personality traits in people with synaesthesia: Do synaesthetes have an atypical personality profile? Pers. Individ. Dif. 54, 828-831.

Barratt, E. L., \& Davis, N. J. (2015). Autonomous Sensory Meridian Response (ASMR): a flow-like mental state. PeerJ, 3, e851.

Bolognini, N., Convento, S., Casati, C., Mancini, F., Brighina, F. and Vallar, G. (2016). Multisensory integration in hemianopia and unilateral spatial neglect: Evidence from the sound induced flash illusion, Neuropsychologia 87, 134-143.

Cecere, R., Rees, G. and Romei, V. (2015). Individual differences in alpha frequency drive crossmodal illusory 
perception, Curr. Biol. 25, 231-235.

Chan, Y. M., Pianta, M. J. and McKendrick, A. M. (2014). Reduced audiovisual recalibration in the elderly, Front. Aging Neurosci. 6, 226. doi: 10.3389/fnagi.2014.00226

Ding, C., Palmer, C. J., Hohwy, J., Youssef, G. J., Paton, B., Tsuchiya, N., Stout, J. C. and Thyagarajan, D. (2017). Parkinson's disease alters multisensory perception: Insights from the Rubber Hand Illusion, Neuropsychologia 97, 38-45.

Ernst, M. O. and Banks, M. S. (2002). Humans integrate visual and haptic information in a statistically optimal fashion, Nature 415(6870), 429-433.

Foss-Feig, J. H., Kwakye, L. D., Cascio, C. J., Burnette, C. P., Kadivar, H., Stone, W. L. and Wallace, M. T. (2010). An extended multisensory temporal binding window in autism spectrum disorders, Exp. Brain Res. 203, 381-389.

Foxe, J. J., Molholm, S., Del Bene, V. A., Frey, H. P., Russo, N. N., Blanco, D., Saint-Amour, D. and Ross, L. A. (2015). Severe multisensory speech integration deficits in high-functioning school-aged children with autism spectrum disorder (ASD) and their resolution during early adolescence, Cereb. Cortex 25, 298312.

Ghazanfar, A. A. and Schroeder, C.E. (2006) Is neocortex essentially multisensory? Trends Cogn. Sci. 10, 278285.

Gori, M., Del Viva, M., Sandini, G. and Burr, D. C. (2008). Young children do not integrate visual and haptic form information, Curr. Biol. 18, 694-698.

Haigh, A., Brown, D. J., Meijer, P. and Proulx, M. J. (2013). How well do you see what you hear? The acuity of visual-to-auditory sensory substitution, Front. Psychol. 4, 330. doi: 10.3389/fpsyg.2013.00330

Kaganovich, N., Schumaker, J., Leonard, L. B., Gustafson, D. and Macias, D. (2014). Children with a history of SLI show reduced sensitivity to audiovisual temporal asynchrony: An ERP study, J. Speech, Lang. Hear. Res. 57, 1480-1502.

Lacey, S., Martinez, M., McCormick, K. and Sathian, K. (2016). Synesthesia strengthens sound-symbolic crossmodal correspondences, Eur. J. Neurosci. 44, 2716-2721.

Lewkowicz, D. J. and Ghazanfar, A. A. (2009). The emergence of multisensory systems through perceptual narrowing, Trends Cogn. Sci. 13, 470-478.

Marotta, A., Bombieri, F., Zampini, M., Schena, F., Dallocchio, C., Fiorio, M. and Tinazzi, M. (2017). The moving rubber hand illusion reveals that explicit sense of agency for tapping movements is preserved in functional movement disorders, Front. Hum. Neurosci. 11, 291. doi: 10.3389/fnhum.2017.00291

Meijer, P. B. (1992). An experimental system for auditory image representations, IEEE Trans. Biomed. Eng. 39, $112-121$.

Peterzell, D. H. and Kennedy, J. F. (2016). Discovering sensory processes using individual differences: A review and factor analytic manifesto, Electron Imaging 2016, 1-11. doi: 10.2352/ISSN.24701173.2016.16HVEI-112

Setti, A., Burke, K. E., Kenny, R. A. and Newell, F. N. (2011). Is inefficient multisensory processing associated with falls in older people? Exp. Brain Res. 209, 375-384. 
Shams, L., Kamitani, Y. and Shimojo, S. (2000). Illusions: What you see is what you hear, Nature 408(6814), 788.

Smith, B. C. (2015). The chemical senses, in: The Oxford Handbook of Philosophy of Perception, M. Matthen (Ed.), pp. 314-352, Oxford University Press, Oxford, UK.

Szycik, G. R., Münte, T. F., Dillo, W., Mohammadi, B., Samii, A., Emrich, H. M. and Dietrich, D. E. (2009). Audiovisual integration of speech is disturbed in schizophrenia: An fMRI study, Schizophr. Res. 110, 111-118.

White, R. C. and Aimola Davies, A. M. (2017). Asynchrony in the rubber hand paradigm: Unexpected illusions following stroke, Cortex 93, 224-226.

Williams, L. E., Light, G. A., Braff, D. L. and Ramachandran, V. S. (2010). Reduced multisensory integration in patients with schizophrenia on a target detection task, Neuropsychologia 48, 3128-3136. 